\title{
ON THE STRATIFICATION OF NONCOMMUTATIVE PRIME SPECTRA
}

\author{
MARTIN LORENZ
}

(Communicated by Binge Huisgen-Zimmermann)

\begin{abstract}
We study rational actions of an algebraic torus $G$ by automorphisms on an associative algebra $R$. The $G$-action on $R$ induces a stratification of the prime spectrum Spec $R$ which was introduced by Goodearl and Letzter. For a noetherian algebra $R$, Goodearl and Letzter showed that the strata of Spec $R$ are isomorphic to the spectra of certain commutative Laurent polynomial algebras. The purpose of this note is to give a new proof of this result which works for arbitrary algebras $R$.
\end{abstract}

\section{INTRODUCTION}

Let $G$ be an affine algebraic group and let $R$ be an associative algebra on which $G$ acts rationally by algebra automorphisms. The induced $G$-action on the set $\operatorname{Spec} R$ of all prime ideals of $R$ leads to a stratification of $\operatorname{Spec} R$ which was pioneered by Goodearl and Letzter [3]. For the special case of an algebraic torus $G$ and a noetherian algebra $R$, Goodearl and Letzter have given a description of the strata of $\operatorname{Spec} R$ in terms of the spectra of certain (commutative) Laurent polynomial algebras. Later, a different description of the strata was given in [6], for any algebra $R$ and any connected affine algebraic group $G$. The purpose of this short note is to consolidate the description of [6], for the case of an algebraic torus $G$, with the earlier one due to Goodearl and Letzter, still working with a general algebra $R$.

Throughout, $\mathbb{k}$ will be an algebraically closed base field of arbitrary characteristic. The largest $G$-stable ideal of the algebra $R$ that is contained in a given ideal $I$ of $R$, called the $G$-core of $I$, will be denoted by $I: G$; so $I: G=\bigcap_{g \in G} g . I$. It is easy to see [5. Proposition 8(b)] that, for any rational action of an affine algebraic group $G$ on $R$, the collection of all $G$-cores of prime ideals of $R$ coincides with the set of all $G$-prime ideals of $R$; the latter set will be denoted by $G$-Spec $R$. If the algebraic group $G$ is connected, then $G$-Spec $R$ is simply the set of all $G$-stable prime ideals of $R$ [5, Proposition 19(a)]. The Goodearl-Letzter stratification of $\operatorname{Spec} R$ is the partition

$$
\operatorname{Spec} R=\bigsqcup_{I \in G-\operatorname{Spec} R} \operatorname{Spec}_{I} R \quad \text { with } \quad \operatorname{Spec}_{I} R=\{P \in \operatorname{Spec} R \mid P: G=I\} .
$$

Received by the editors June 5, 2012 and, in revised form, September 26, 2012.

2010 Mathematics Subject Classification. Primary 16W22, 17B37, 20G42.

Key words and phrases. Algebraic group, rational action, algebraic torus, rational ideal, prime spectrum, stratification.

The research of the author was supported in part by NSA Grant H98230-12-1-0221. 
For an algebraic torus $G$, we will give a description of each stratum $\operatorname{Spec}_{I} R$ in terms of the spectrum of a suitable affine commutative algebra $Z_{I}$. The construction of the algebra $Z_{I}$ and the precise statement of the main result will be given in Section 1, while the proof will occupy Section 2

\section{The Stratification Theorem}

Let $G$ be a connected affine algebraic group over $\mathbb{k}$ and let $R$ be an associative $\mathbb{k}$-algebra with a rational $G$-action by $\mathbb{k}$-algebra automorphisms. For a given $I \in$ $G$-Spec $R$, let $\mathcal{C}(R / I)$ denote the extended centroid of the algebra $R / I$; this is a $\mathbb{k}$-field, called the heart of $I$, on which $G$ acts via its action on $R / I$ [5, 2.3]. We put $Z_{I}=\{c \in \mathcal{C}(R / I) \mid$ the orbit $G . c$ spans a finite-dimensional $\mathbb{k}$-subspace of $\mathcal{C}(R / I)\}$.

Clearly, $Z_{I}$ is a $G$-stable $\mathbb{k}$-subalgebra of $\mathcal{C}(R / I)$ and $Z_{I}$ contains the subfield of $G$-invariants, $\mathcal{C}(R / I)^{G}$. Moreover, the $G$-action on $Z_{I}$ is rational by [5, Lemma $18(\mathrm{~b})]$.

We now focus on the case of an algebraic torus $G$. As usual, $X(G)$ will denote the lattice of rational characters of $G$. The following theorem, under the additional assumption that the algebra $R$ is noetherian, is originally due to Goodearl and Letzter [3]; see also [2], 4] and [1, II.2.13].

Stratification Theorem. Let $G$ be an algebraic torus over $\mathbb{k}$ that acts rationally by algebra automorphisms on the $\mathbb{k}$-algebra $R$, and let $I \in G$-Spec $R$. Then:

(a) There is an isomorphism

$$
Z_{I} \cong \mathcal{C}(R / I)^{G} \Gamma_{I},
$$

the group algebra over the field $\mathcal{C}(R / I)^{G}$ of the sublattice

$$
\Gamma_{I}=X\left(G / \operatorname{Ker}_{G}\left(Z_{I}\right)\right) \subseteq X(G) .
$$

(b) There is a G-equivariant order isomorphism

$$
\gamma: \operatorname{Spec}_{I} R \stackrel{\sim}{\longrightarrow} \operatorname{Spec}\left(Z_{I}\right) .
$$

\section{Proof of the Stratification Theorem}

2.1. Reductions and preliminaries. We begin with some remarks that hold for an arbitrary connected affine algebraic group $G$ over $\mathbb{k}$. In order to describe the $G$-stratum $\operatorname{Spec}_{I} R$ for a given $I \in G$-Spec $R$, we may replace $R$ by $R / I$ and thus assume that $I=0$. In particular, $R$ is a prime ring. We will write $\mathcal{C}=\mathcal{C}(R)$ and $Z=Z_{0}$ for brevity; so $\mathcal{C}$ is a commutative $\mathbb{k}$-field on which $G$ acts by automorphisms and

$$
Z=\{c \in \mathcal{C} \mid \text { the orbit } G . c \text { spans a finite-dimensional } \mathbb{k} \text {-subspace of } \mathcal{C}\} .
$$

Our goal is to give a description of $Z$ and to establish a suitable order isomorphism

$$
\gamma: \operatorname{Spec}_{0} R=\{P \in \operatorname{Spec} R \mid P: G=0\} \stackrel{\sim}{\longrightarrow} \operatorname{Spec}(Z) .
$$

We will need to consider various $G$-actions; they will usually be indicated by a simple dot as in the foregoing. When more precision is necessary, the $G$-action on $\mathcal{C}$ will be denoted by $\rho$. The group $G$ also acts on $\mathbb{k}[G]$, the algebra of regular functions 
of $G$, via the right and left regular representations $\rho_{r}, \rho_{\ell}: G \rightarrow \operatorname{Aut}_{\mathbb{k} \text {-alg }}(\mathbb{k}[G])$; they are defined by $\left(\rho_{r}(x) f\right)(y)=f(y x)$ and $\left(\rho_{\ell}(x) f\right)(y)=f\left(x^{-1} y\right)$ for $x, y \in G$ and $f \in \mathbb{k}[G]$.

2.2. The algebra $Z$. Consider the $\operatorname{Hopf} \mathcal{C}$-algebra

$$
S=\mathcal{C} \otimes_{\mathbb{k}} \mathbb{k}[G]
$$

this is an algebra of $\mathcal{C}$-valued functions on $G$ via $\left(\sum_{i} c_{i} \otimes f_{i}\right)(g)=\sum_{i} c_{i} f_{i}(g)$. The group $G$ acts on the ring $S$ via $\rho \otimes \rho_{r}$. If $g \in G$ and $s=\sum_{i} c_{i} \otimes f_{i} \in S$, then g.s $\in S$ is the function $G \rightarrow \mathcal{C}$ that is given by

$$
(g . s)(x)=\sum_{i}\left(g \cdot c_{i}\right) f_{i}(x g)=g \cdot \sum_{i} c_{i} f_{i}(x g) \quad(x \in G) .
$$

Let $S^{G}$ denote the subring of $G$-invariants in $S$. Thus, $s \in S^{G}$ if and only if $g^{-1} . s(1)=s(g)$ for all $g \in G$.

We claim that

$$
S^{G} \subseteq Z \otimes_{\mathbb{k}} \mathbb{k}[G] .
$$

To see this, let $s=\sum_{1}^{r} c_{i} \otimes f_{i} \in S^{G}$ with $\left\{f_{i}\right\}_{1}^{r} \subseteq \mathbb{k}[G]$ chosen $\mathbb{k}$-linearly independent. For a given $g \in G$, choose $\left\{x_{j}\right\}_{i}^{r} \subseteq G$ such that the matrix $A_{g}=\left(f_{i}\left(x_{j} g\right)\right)_{i, j}$ is invertible and put $B=\left(f_{i}\left(x_{j}\right)\right)_{i, j}$. Then the equations $\sum_{i} c_{i} f_{i}(x)=\sum_{i}\left(g . c_{i}\right) f_{i}(x g)$ for all $x \in G$, with $x=x_{j}$ for $j=1, \ldots, r$, can be written as the matrix equation $\left(c_{1}, \ldots, c_{r}\right) B=\left(g \cdot c_{1}, \ldots, g \cdot c_{r}\right) A_{g}$ or else $\left(g . c_{1}, \ldots, g \cdot c_{r}\right)=\left(c_{1}, \ldots, c_{r}\right) B A_{g}^{-1}$. This shows that $G . c_{i} \subseteq \sum_{i=1}^{r} \mathbb{k} c_{i}$ and hence $c_{i} \in Z$, proving (10).

Next, we show that there is an isomorphism

$$
\varepsilon: S^{G} \stackrel{\sim}{\longrightarrow} Z
$$

such that

$$
\varepsilon \circ\left(1_{Z} \otimes \rho_{l}(g)\right)=\rho(g) \circ \varepsilon
$$

holds for all $g \in G$. Indeed, by [5, Lemma 18(b)], the $G$-action on $Z$ is rational: it arises from a map of $\mathbb{k}$-algebras $\Delta_{Z}: Z \rightarrow Z \otimes_{\mathbb{k}} \mathbb{k}[G], c \mapsto \sum c_{0} \otimes c_{1}$, via $g . c=\sum c_{0} c_{1}(g)$. By [6, equations (17) and (18)], the $\mathbb{k}[G]$-linear extension of $\Delta_{Z}$, which will also be denoted by $\Delta_{Z}$, is an isomorphism of $\mathbb{k}[G]$-algebras,

$$
\Delta_{Z}: Z \otimes_{\mathbb{k}} \mathbb{k}[G] \stackrel{\sim}{\longrightarrow} Z \otimes_{\mathbb{k}} \mathbb{k}[G],
$$

that satisfies the "intertwining formula" $\Delta_{Z} \circ\left(\rho \otimes \rho_{r}\right)(g)=\left(1_{Z} \otimes \rho_{r}\right)(g) \circ \Delta_{Z}$ for each $g \in G$. It follows that $\Delta_{Z}$ yields an isomorphism of $S^{G}=\left(Z \otimes_{\mathbb{k}} \mathbb{k}[G]\right)^{G}$ with the subalgebra of $\left(1_{Z} \otimes \rho_{r}\right)(G)$-invariants in $Z \otimes_{\mathbb{k}} \mathbb{k}[G]$. Since the latter algebra is clearly $Z$, the isomorphism (2) follows. It is easy to see that the isomorphism (2) is just the restriction to $S^{G}$ of the Hopf counit $S \rightarrow \mathcal{C}, s \mapsto s(1)$. In particular, for $s \in S^{G}$, we have $\left(\varepsilon \circ\left(1_{Z} \otimes \rho_{l}(g)\right)\right)(s)=s\left(g^{-1}\right)=g . s(1)=(\rho(g) \circ \varepsilon)(s)$, proving (3)).

2.3. The case of an algebraic torus. Now let $G \cong\left(\mathbb{k}^{\times}\right)^{d}$ be an algebraic torus over $\mathbb{k}$ and let $\Lambda=X(G) \cong \mathbb{Z}^{d}$ be its lattice of rational characters. Then $\mathbb{k}[G]=\mathbb{k} \Lambda$, the group algebra of $\Lambda$ over $\mathbb{k}$. As it is customary to use additive notation for the lattice $\Lambda$, we will write the standard $\mathbb{k}$-basis of $\mathbb{k}[G]$ as $\left\{\mathbf{x}^{\lambda} \mid \lambda \in \Lambda\right\}$; so $\mathbf{x}^{\lambda} \mathbf{x}^{\lambda^{\prime}}=\mathbf{x}^{\lambda+\lambda^{\prime}}$ and $\mathbf{x}^{\lambda}(g)=\langle\lambda, g\rangle \in \mathbb{k}^{\times}$for $g \in G$. Then $\rho_{r}(g) \mathbf{x}^{\lambda}=\langle\lambda, g\rangle \mathbf{x}^{\lambda}$ and

$$
S=\bigoplus_{\lambda \in \Lambda} \mathcal{C} \otimes_{\mathbb{k}} \mathbb{k} \mathbf{x}^{\lambda} \cong \mathcal{C} \Lambda,
$$


the group algebra of $\Lambda$ over the field $\mathcal{C}$. Consider an element $s=\sum_{\lambda} s_{\lambda} \otimes \mathbf{x}^{\lambda} \in S$ with $s_{\lambda} \in \mathcal{C}$. Then $g . s=\sum_{\lambda} g . s_{\lambda} \otimes\langle\lambda, g\rangle \mathbf{x}^{\lambda}$ for $g \in G$. Hence, $s \in S^{G}$ if and only if $g . s_{\lambda}=\langle-\lambda, g\rangle s_{\lambda}$ for all $g, \lambda$. Putting $\mathcal{C}_{\lambda}=\{c \in \mathcal{C} \mid g . c=\langle\lambda, g\rangle c$ for all $g \in G\}$ and noting that each nonzero $\mathcal{C}_{\lambda}$ is 1 -dimensional over the fixed field $\mathcal{C}^{G}$, we have

$$
S^{G}=\bigoplus_{\lambda \in \Lambda} \mathcal{C}_{-\lambda} \otimes_{\mathbb{k}} \mathbb{k} \mathbf{x}^{\lambda} \cong \mathcal{C}^{G} \Gamma
$$

the group algebra of the sublattice $\Gamma=\left\{\lambda \in \Lambda \mid \mathcal{C}_{\lambda} \neq 0\right\}$ over $\mathcal{C}^{G}$. We remark that $\Gamma=X(G / N)$ is the character lattice of the torus $G / N$, where $N$ is the kernel of the action of $G$ on $Z$; so $Z=\bigoplus_{\lambda \in \Gamma} \mathcal{C}_{\lambda}$. The isomorphisms (4) and (2) prove part (a) of the theorem. Note also that $S$ is free over $S^{G}$.

We claim that each $G$-stable ideal $\mathfrak{a}$ of $S$ is generated by its intersection with $S^{G}$ :

$$
\mathfrak{a}=\left(\mathfrak{a} \cap S^{G}\right) S .
$$

For the nontrivial inclusion $\subseteq$, let $s=\sum_{\lambda} s_{\lambda} \otimes \mathbf{x}^{\lambda} \in \mathfrak{a}$ be given with $s_{\lambda} \in \mathcal{C}$. In order to show that $s \in\left(\mathfrak{a} \cap S^{G}\right) S$, we argue by induction on the size of $\operatorname{Supp}(s)=\{\lambda \in$ $\left.\Lambda \mid s_{\lambda} \neq 0\right\}$, the length of $s$. Our claim being clear for $s=0$, assume that $s \neq 0$. Suppose there exists an element $0 \neq t \in \mathfrak{a}$ with $\operatorname{Supp}(t) \varsubsetneqq \operatorname{Supp}(s)$. Multiplying $t$ and $s$ with suitable units of the form $c \otimes \mathbf{x}^{\mu}$, we may assume that $0 \in \operatorname{Supp}(t)$ and $t_{0}=s_{0}=1$. Since $t$ and $s-t$ are shorter than $s$, they both belong to $\left(\mathfrak{a} \cap S^{G}\right) S$ by induction, and hence $s \in\left(\mathfrak{a} \cap S^{G}\right) S$ as well. Therefore, we may assume that if $t \in \mathfrak{a}$ and $\operatorname{Supp}(t) \varsubsetneqq \operatorname{Supp}(s)$, then $t=0$. Continuing to assume that $s_{0}=1$, this applies in particular to $t=s-g . s=\sum_{0 \neq \lambda}\left(s_{\lambda}-\langle\lambda, g\rangle g . s_{\lambda}\right) \otimes \mathbf{x}^{\lambda}$ for each $g \in G$. Therefore, $s \in S^{G}$ and (5) is proved.

Now let $\mathfrak{b}$ be an ideal of $S^{G}$ and let $\mathfrak{a}$ denote sum of all ideals of $S$ that contract to $\mathfrak{b}$. Since $S$ is free over $S^{G}$, we have $\mathfrak{a} \cap S^{G}=\mathfrak{b}$. Moreover, $\mathfrak{a}$ is clearly $G$-stable and so (5) gives that $\mathfrak{a}=\mathfrak{b} S$. Thus, $\mathfrak{b} S$ is the unique largest ideal of $S$ that contracts to $\mathfrak{b}$.

2.4. The prime correspondence. We start with some reminders from [6]. For now, let $G$ again be an arbitrary connected affine algebraic group over $\mathbb{k}$ and let $\mathbb{k}(G)=$ Fract $\mathbb{k}[G]$ be the field of rational functions of $G$. The $G$-action on $S$ via $\rho \otimes \rho_{r}$ extends uniquely to an action of $G$ on the following localization of $S$ :

$$
T=\mathcal{C} \otimes_{\mathbb{k}} \mathbb{k}(G) .
$$

Let $\operatorname{Spec}^{G}(T)$ denote the collection of all $G$-stable prime ideals of $T$. Then 6 , Theorem 9] establishes an order isomorphism

$$
c: \operatorname{Spec}_{0} R \stackrel{\sim}{\longrightarrow} \operatorname{Spec}^{G}(T)
$$

with the following $G$-equivariance property, for $P \in \operatorname{Spec}_{0} R$ and $g \in G$ :

$$
c(g . P)=\left(1_{\mathcal{C}} \otimes \rho_{\ell}(g)\right)(c(P)) .
$$

Since $T$ is the localization $S$ at the nonzero elements of $\mathbb{k}[G]$, contraction and extension yields a $G$-equivariant order isomorphism

$$
\operatorname{Spec} T \stackrel{\sim}{\longrightarrow}\{\mathfrak{p} \in \operatorname{Spec} S \mid \mathfrak{p} \cap \mathbb{k}[G]=0\} .
$$


Note that $\mathbb{k}[G]$ is a $G$-simple ring, because $G$ acts transitively on itself by right multiplication. Therefore, each $\mathfrak{p} \in \operatorname{Spec}^{G}(S)$ satisfies $\mathfrak{p} \cap \mathbb{k}[G]=0$, and hence the above bijection restricts to a bijection

$$
\operatorname{Spec}^{G}(T) \stackrel{\sim}{\longrightarrow} \operatorname{Spec}^{G}(S)
$$

given by contraction and extension.

We now return to the case of an algebraic torus $G$. Then the map $\mathfrak{p} \mapsto \mathfrak{p} \cap S^{G}$ injects $\operatorname{Spec}^{G}(S)$ into $\operatorname{Spec}\left(S^{G}\right)$ by (5). Moreover, for any $\mathfrak{q} \in \operatorname{Spec}\left(S^{G}\right)$, we know that $\mathfrak{p}=\mathfrak{q} S$ is the unique largest ideal of $S$ that contracts to $\mathfrak{q}$, which implies that $\mathfrak{p} \in \operatorname{Spec}^{G}(S)$. Thus we obtain a bijection

$$
\operatorname{Spec}^{G}(S) \stackrel{\sim}{\longrightarrow} \operatorname{Spec}\left(S^{G}\right)
$$

that is again given by contraction and extension. From (6) - (9) in conjunction with the isomorphism (2) we obtain the desired order isomorphism $\gamma: \operatorname{Spec}_{0} R \stackrel{\sim}{\longrightarrow}$ $\operatorname{Spec}(Z)$. The fact that $\gamma$ is $G$-equivariant is immediate from the $G$-equivariance property (7) of the bijection (6) and the intertwining formula (3). This completes the proof of the Stratification Theorem.

\section{ACKNOWLEDGMENTS}

The author would like to thank Ken Goodearl for an invitation to the University of California, Santa Barbara, in the spring of 2012 and for many fruitful discussions during that visit. Thanks are also due to the referee for an insightful report.

\section{REFERENCES}

[1] Ken A. Brown and Ken R. Goodearl, Lectures on algebraic quantum groups, Advanced Courses in Mathematics. CRM Barcelona, Birkhäuser Verlag, Basel, 2002. MR1898492 (2003f:16067)

[2] K. R. Goodearl, Prime spectra of quantized coordinate rings, Interactions between ring theory and representations of algebras (Murcia), Lecture Notes in Pure and Appl. Math., vol. 210, Dekker, New York, 2000, pp. 205-237. MR.1759846 (2001b:16052)

[3] K. R. Goodearl and E. S. Letzter, The Dixmier-Moeglin equivalence in quantum coordinate rings and quantized Weyl algebras, Trans. Amer. Math. Soc. 352 (2000), no. 3, 1381-1403, DOI 10.1090/S0002-9947-99-02345-4. MR1615971 (2000j:16040)

[4] K. R. Goodearl and J. T. Stafford, The graded version of Goldie's theorem, Algebra and its applications (Athens, OH, 1999), Contemp. Math., vol. 259, Amer. Math. Soc., Providence, RI, 2000, pp. 237-240, DOI 10.1090/conm/259/04098. MR1780524 (2001g:16087)

[5] Martin Lorenz, Group actions and rational ideals, Algebra Number Theory 2 (2008), no. 4, 467-499, DOI 10.2140/ant.2008.2.467. MR2411408(2009i:16058)

[6] Martin Lorenz, Algebraic group actions on noncommutative spectra, Transform. Groups 14 (2009), no. 3, 649-675, DOI 10.1007/s00031-009-9059-8. MR2534802 (2010m:14060)

Department of Mathematics, Temple University, Philadelphia, Pennsylvania 19122

E-mail address: lorenz@temple.edu

$U R L:$ http://www . math.temple.edu/ lorenz 